BMJ Open

Diabetes

Research

\& Care

\title{
Association of severe hypoglycemia with depressive symptoms in patients with type 2 diabetes: the Fukuoka Diabetes Registry
}

\author{
Yohei Kikuchi, ${ }^{1}$ Masanori Iwase, ${ }^{1,2}$ Hiroki Fujii, ${ }^{3}$ Toshiaki Ohkuma, ${ }^{4}$ Shinako Kaizu, ${ }^{1}$ \\ Hitoshi Ide, ${ }^{1}$ Tamaki Jodai, ${ }^{1}$ Yasuhiro Idewaki, ${ }^{2}$ Udai Nakamura, ${ }^{1}$ \\ Takanari Kitazono ${ }^{1}$
}

To cite: Kikuchi Y, Iwase M, Fujii $\mathrm{H}$, et al. Association of severe hypoglycemia with depressive symptoms in patients with type 2 diabetes: the Fukuoka Diabetes Registry. BMJ Open Diabetes Research and Care 2015;3: e000063. doi:10.1136/ bmjdrc-2014-000063

Received 26 September 2014 Revised 23 March 2015 Accepted 1 April 2015

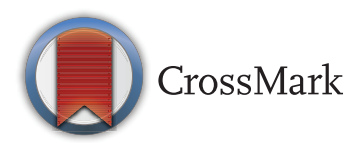

${ }^{1}$ Department of Medicine and Clinical Science, Graduate School of Medical Sciences, Kyushu University, Fukuoka, Japan

${ }^{2}$ Diabetes Center, Hakujyuji Hospital, Fukuoka, Japan ${ }^{3}$ Division of General Internal Medicine, School of Oral Health Science, Kyushu Dental University, Kitakyushu, Japan

${ }^{4}$ Division of Research Management, Center for Cohort Studies, Graduate School of Medical Sciences, Kyushu University, Fukuoka, Japan

\section{Correspondence to}

Dr Masanori Iwase;

iwase@intmed2.med.kyushuu.ac.jp

\section{ABSTRACT}

Objective: Although many studies have investigated the clinical characteristics of patients with diabetes with depression in Western populations, there is a lack of information regarding other ethnicities. We studied the association between clinical characteristics and depressive symptoms in Japanese patients with type 2 diabetes.

Methods: A total of 4218 Japanese patients with type 2 diabetes who were not taking antidepressants were divided into four groups according to the Center for Epidemiologic Studies Depression Scale (CES-D) score. The relationship between the severity of depressive symptoms and clinical parameters was examined cross-sectionally.

Results: After multivariate adjustments, the severity of depressive symptoms was significantly associated with body mass index, leisure-time physical activity, current smoking, sleep duration, sucrose intake, skipping breakfast, insulin use, severe hypoglycemia, dysesthesia of both feet, history of foot ulcer, photocoagulation, ischemic heart disease, and stroke. ORs for severe hypoglycemia increased significantly with the CES-D score in 2756 sulfonylurea and/or insulin-treated patients after multivariate adjustment including age, sex, duration of diabetes, glycated hemoglobin, insulin use, self-monitoring of blood glucose, leisure-time physical activity, skipping breakfast, dysesthesia of both feet, ischemic heart disease, and stroke (CES-D score $\leq 9$, referent; 10-15, OR 1.64; 16-23, OR 2.09; $\geq 24$, OR 3.66; $p$ for trend $<0.01$ ).

Conclusions: Severe hypoglycemia was positively associated with the severity of depressive symptoms in Japanese patients with type 2 diabetes independent of glycemic control, insulin therapy, lifestyle factors, and diabetic complications. As both severe hypoglycemia and depression are known risk factors for morbidity and mortality in patients with diabetes, clinicians should be aware of this association.

UMIN Clinical Trial Registry: 000002627.

\section{INTRODUCTION}

Depression and diabetes mellitus frequently coexist, ${ }^{1}{ }^{2}$ and a bidirectional relationship

\section{Key messages}

- We confirmed clinical characteristics of depressed Japanese patients with diabetes similar to those reported in Western populations, that is, obesity, unhealthy lifestyles, poor glycemic control, and advanced diabetic complications.

- Severe hypoglycemia was significantly associated with the severity of depressive symptoms after adjusting for confounding factors.

- Severe hypoglycemia and depression are known risk factors for morbidity and mortality in patients with diabetes, clinicians should be aware of this combination in clinical settings.

has been reported. ${ }^{3}{ }^{4}$ Depression worsens glycemic control, ${ }^{5}$ accelerates the development and progression of diabetic complications, and leads to increased mortality. ${ }^{6} \quad 7$ Many studies have investigated the clinical characteristics of patients with diabetes with depression in Western populations. However, since the development of depression is influenced by cultural and societal factors, studies in other ethnic groups are important. This is particularly the case in East Asia, where the prevalence of type 2 diabetes is increasing rapidly ${ }^{9}$ and suicide rates are very high. For example, in 2012, annual suicide rates in Korea and Japan were 28.9 and 18.5 per 100000 , respectively, making them the first and fourth highest among developed countries. ${ }^{10}$

In the present study, we aimed to build on research in Western populations and extend understanding of the relationship between depression and type 2 diabetes to East Asians. Therefore, we investigated the relationship of the severity of depressive symptoms with clinical parameters, including an episode of severe hypoglycemia, in Japanese patients with type 2 diabetes. 


\section{METHODS}

\section{Study participants}

The Fukuoka Diabetes Registry is a multicenter prospective study designed to investigate the influence of modern treatments on prognosis in patients with diabetes regularly attending teaching hospitals certified by the Japan Diabetes Society or certified diabetologists' clinics in Fukuoka Prefecture, Japan (UMIN Clinical Trial Registry 000002627).${ }^{11}$ A total of 5131 patients with diabetes aged $\geq 20$ years were registered between April 2008 and October 2010. Exclusion criteria were: (1) patients with drug-induced diabetes or receiving corticosteroid treatment; (2) patients undergoing renal replacement therapy; (3) patients with serious diseases other than diabetes, such as advanced malignancy or decompensated liver cirrhosis; and (4) patients unable to visit a diabetologist regularly. Patients with type 1 diabetes mellitus (negative serum C peptide and/or positive antiglutamic acid decarboxylase antibody), those who had already eaten breakfast, and those who took antidepressants or antipsychotic drugs were excluded. In total, 4218 (2408 men and 1810 women) were enrolled in this cross-sectional study. The study was conducted with the approval of the Kyushu University Institutional Review Board, and written informed consent was obtained from all participants.

\section{Clinical evaluation}

Participants completed a self-administered questionnaire to collect information on diabetes duration, alcohol intake, smoking habits, sleep duration, physical activity, and severe hypoglycemia during the previous year. Severe hypoglycemia was defined as an episode of hypoglycemia requiring assistance for recovery. Body weight and height were measured, body mass index (BMI) was calculated as weight $(\mathrm{kg})$ divided by height squared $\left(\mathrm{m}^{2}\right)$ and obesity was defined according to the Japan Society for the Study of Obesity thresholds as a BMI $\geq 25 \mathrm{~kg} / \mathrm{m}^{2}$. ${ }^{12}$ Waist circumference was measured at the umbilical level, with the participant in the standing position, by a trained staff member. Blood pressure was measured with the participant in the sitting position. Participants' medical records were reviewed for all medications including antidepressants and antipsychotic drugs. Leisure-time physical activity information was obtained using a self-reported questionnaire, and metabolic equivalent (met) hours per week was calculated using Ainsworth's methods. ${ }^{13}$ The dietary survey was conducted using a brief-type self-administered diet history questionnaire regarding the food frequency of 58 items (BDHQ; Gender Medical Research Inc, Tokyo, Japan). The validity of ranking the energy-adjusted intakes of many nutrients has been previously studied in an adult Japanese population. ${ }^{14}$ The presence of depressive symptoms was assessed using the Center for Epidemiologic Studies Depression Scale (CES-D).${ }^{15}$ The CES-D consists of 20 items and scores range from 0 to 60. Higher scores indicate more severe depressive symptoms, with a score of 9 as an average in the general community, ${ }^{15} 16$ as a general cut-off, ${ }^{15}$ and 24 as a more conservative cut-off. ${ }^{16}$ We divided the participants into four groups according to the CES-D score as follows: $\leq 9 \quad(\mathrm{n}=3327) ; 10-15 \quad(\mathrm{n}=539) ; 16-23 \quad(\mathrm{n}=240)$; and $\geq 24 \quad(\mathrm{n}=112)$.

\section{Laboratory measurements}

Blood was collected by venipuncture after an overnight fast, and spot urine samples were obtained. Assessments were performed at one central laboratory (Kyushu University Hospital, Fukuoka, Japan). Glycated hemoglobin (HbA1c) was determined using high-performance liquid chromatography (Tosoh Corp, Tokyo, Japan); plasma glucose by the glucose oxidase method; serum C peptide and folate by chemiluminescent immunoassay (Kyowa Medex, Tokyo, Japan; Siemens Healthcare Diagnostics, Tokyo, Japan); serum adiponectin and high sensitivity C reactive protein (HS-CRP) by latex immunonephelometry (Mitsubishi Chemical Medience, Tokyo, Japan; Siemens Healthcare Diagnostics, Tokyo, Japan); urinary albumin by immunonephelometry (Medical and Biological Laboratories, Nagoya, Japan); and serum total cholesterol, low-density lipoprotein (LDL) cholesterol, high-density lipoprotein (HDL) cholesterol, triglyceride, creatinine, magnesium, and urine creatinine by enzymatic methods. Estimated glomerular filtration rate (eGFR) was calculated using the equation proposed by the Japanese Society of Nephrology. ${ }^{17}$ Albuminuria was defined as urinary albumin excretion $\geq 30 \mathrm{mg} / \mathrm{g}$ creatinine, and chronic kidney disease was defined as albuminuria and/or eGFR $<60 \mathrm{~mL} / \mathrm{min} / 1.73 \mathrm{~m}^{2}$. ${ }^{18}$ Metabolic syndrome was defined according to the definition of 'Harmonizing the Metabolic Syndrome', ${ }^{19}$ that is, the presence of at least two of the following four components: central obesity for Asians (waist circumference $\geq 90 \mathrm{~cm}$ in males and $\geq 80 \mathrm{~cm}$ in females), elevated triglycerides $(\geq 1.69 \mathrm{mmol} / \mathrm{L}$ and $/$ or the use of triglyceridelowering drugs), reduced HDL cholesterol $(<1.03 \mathrm{mmo} /$ $\mathrm{L}$ in males and $<1.29 \mathrm{mmol} / \mathrm{L}$ in females), and elevated blood pressure (systolic blood pressure $\geq 130 \mathrm{~mm} \mathrm{Hg}$ and/or diastolic blood pressure $\geq 85 \mathrm{~mm} \mathrm{Hg}$ and/or the use of antihypertensive drugs). $\beta$-Cell function and insulin resistance were estimated based on fasting glucose and $\mathrm{C}$ peptide concentrations using the homeostasis model assessment (HOMA) calculator, V.2.2.2., ${ }^{20}$ and expressed as the HOMA $\beta$-cell function (HOMA2\%-B) and the HOMA-insulin resistance (HOMA2-IR), respectively.

\section{Statistical analysis}

HS-CRP and adiponectin were log-transformed for the statistical analyses because of having a skewed distribution. Linear trends were examined using multiple regression analysis, and trends in binomial proportions by the Cochran-Armitage test. Multivariate adjustments included age, sex, duration of diabetes, BMI, HbA1c, insulin use, current smoking, current drinking, leisure- 
time physical activity, and total energy intake. The multivariate-adjusted OR and 95\% CIs for severe hypoglycemia were calculated using a multiple logistic regression model. Multivariate adjustments included age, sex, duration of diabetes, HbAlc, insulin use, self-monitoring of blood glucose, leisure-time physical activity, skipping breakfast, dysesthesia of both feet, ischemic heart disease, and stroke. All analyses were performed using the JMP V.11 (SAS Institute Inc, Cary, North Carolina, USA). p Values $<0.05$ were considered to be statistically significant in all analyses.

\section{RESULTS}

Table 1 shows clinical characteristics of studied participants according to the CES-D score. As the CES-D score increased, the participants became younger and the following parameters increased: the proportion of females, prevalence of positive family history of diabetes, BMI, prevalence of obesity (BMI $\geq 25.0$ ), waist circumference, prevalence of abdominal obesity, HbA1c, HOMA2-IR, prevalence of sulfonylurea users, prevalence of insulin users, self-monitoring of blood glucose, and severe hypoglycemia. On the other hand, the duration of diabetes, fasting serum $\mathrm{C}$ peptide, HOMA2\%-B, adiponectin, HS-CRP, and the prevalence of oral hypoglycemic users did not differ among groups. After adjusting for age and sex, the statistical significance for a family history of diabetes, HOMA2-IR, and the prevalence of sulfonylurea users was abolished. BMI and the prevalence of insulin users and severe hypoglycemia increased according to the CES-D score after multivariate adjustments including age, sex, duration of diabetes, BMI (not used as the confounding factor for BMI), HbAlc, insulin use (not used as the confounding factor for the prevalence of insulin users), current smoking, current drinking, leisure-time physical activity, and total energy intake.

Cardiovascular risk factors and diabetic complications according to the CES-D score are shown in table 2. Triglyceride levels, as well as the prevalence of metabolic syndrome, dysesthesia of both feet, history of foot ulcer, photocoagulation, macroalbuminuria $(\geq 300 \mathrm{mg} / \mathrm{g})$, ischemic heart disease, and stroke, increased as the CES-D scores increased. In contrast, blood pressure, antihypertensive drug use, total cholesterol, LDL cholesterol, HDL cholesterol, statin use, albuminuria $(\geq 30 \mathrm{mg} / \mathrm{g}$ ), chronic kidney disease, and malignancy did not differ among groups. After adjusting for age and sex, the statistical significance for triglyceride levels was abolished. After multivariate adjustments, as described above, the prevalence of dysesthesia of both feet, history of foot ulcer, photocoagulation, stroke, and ischemic heart disease increased with the CES-D score.

Table 3 shows the lifestyle factors according to the CES-D score. The prevalence of current smoking increased with the CES-D score. Leisure-time physical activity, sleep duration, intakes of dietary fiber, vitamin C, magnesium and folate, and serum magnesium concentration decreased according to the CES-D score, whereas sucrose intake increased. The prevalence of skipping breakfast increased with the CES-D score. The prevalence of current drinking, as well as intakes of total energy, protein, fat and carbohydrate, and serum folate level, did not differ among groups. After adjusting for age and sex, the statistical significance for dietary fiber was abolished. After multivariate adjustments as described above, the significance remained for the prevalence of current smoking, leisure-time physical activity, sleep duration, sucrose intake, and the prevalence of skipping breakfast.

Finally, the association of severe hypoglycemia with CES-D score was examined in sulfonylurea and/or insulin-treated patients (table 4). The prevalence of severe hypoglycemia increased according to the CES-D score ( $\mathrm{p}$ for trend $<0.0001$ ). The OR for severe hypoglycemia increased significantly according to the CES-D score ( $p$ for trend $<0.01$ ) by multiple logistic regression analysis after multivariate adjustment, including for age, sex, duration of diabetes, HbA1c, insulin treatment, selfmonitoring of blood glucose, leisure-time physical activity, skipping breakfast, dysesthesia of both feet, ischemic heart disease, and stroke.

\section{DISCUSSION}

This study demonstrated that severe hypoglycemia was significantly associated with the severity of depressive symptoms after adjusting for confounding factors including glycemic control, insulin use, lifestyle factors, and diabetic complications. It has been recently reported that severe hypoglycemia is associated with increased morbidity and mortality in patients with type 2 diabetes, although the mechanisms are not fully understood. ${ }^{21}$ Moreover, depression is associated with poor glycemic control, ${ }^{5}$ obesity, ${ }^{22}$ and unhealthy lifestyle factors such as smoking, ${ }^{22}$ reduced physical activity, ${ }^{23}$ and diet, ${ }^{24}$ leading to the development of diabetic complications and increased mortality. ${ }^{6} 7$ In this context, the association of severe hypoglycemia with depression may have great impact on the prognosis in patients with type 2 diabetes.

Some studies have shown the association between hypoglycemia and depression. In a self-reported survey mailed to 200000 households representative of the US adult population, ${ }^{25}$ moderately severe to severe depression was more frequent in those with self-reported hypoglycemia $(n=627)$ than those without $(n=2091 ; 10 \%$ vs $5 \%, \mathrm{p}<0.001$ ). The association between hypoglycemia and depression remained statistically significant after adjusting for comorbid conditions and insulin use. However, this study was based only on mailed questionnaires. In a longitudinal prospective study in primary care clinics in Western Washington, ${ }^{26} 10.7 \%$ of depressed patients with diabetes $(n=495)$ had one or more episodes of severe hypoglycemia compared with $6.4 \%$ of non-depressed patients $(n=3622)$ over the 5 -year 


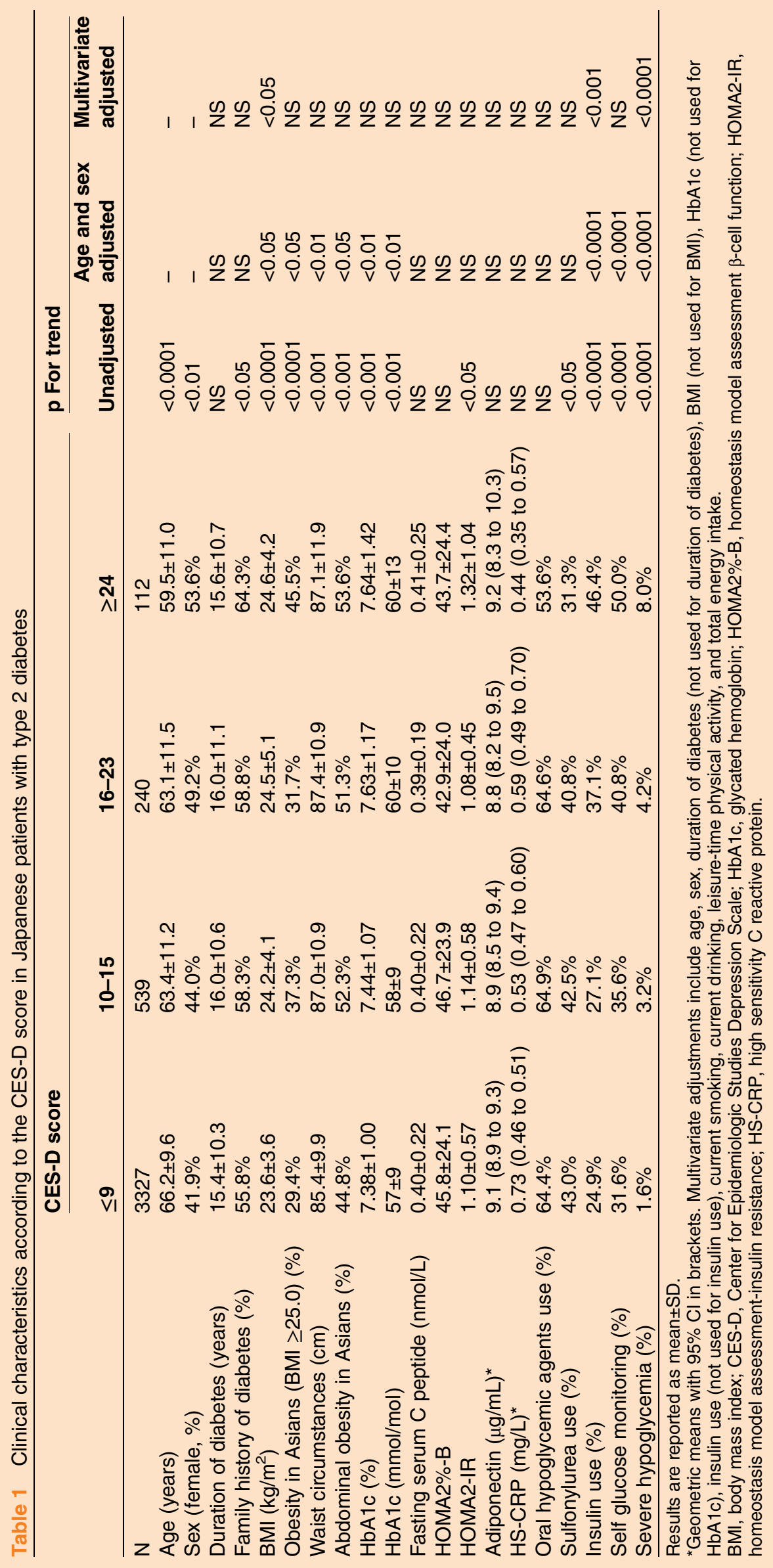




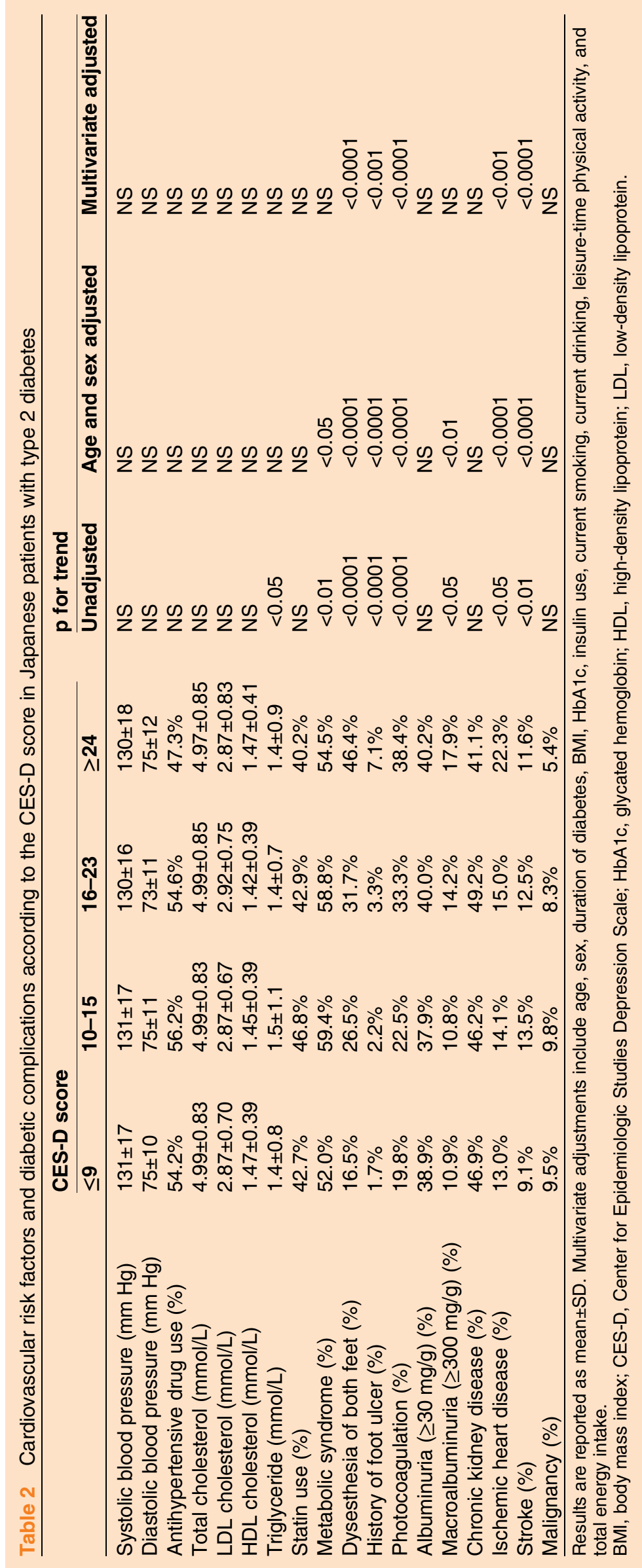




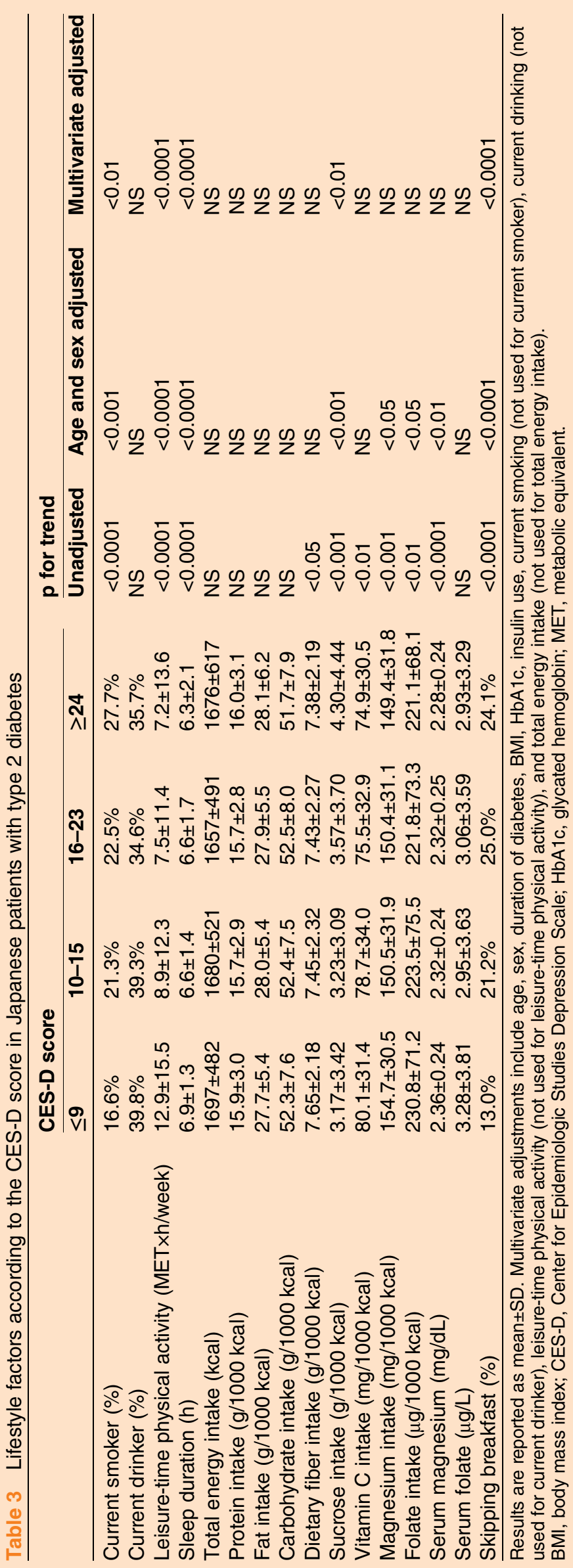

follow-up period. Depressed patients had a significantly higher risk of developing severe hypoglycemia (HR 1.42) and a greater number of episodes of severe hypoglycemia (OR 1.34) after multivariate adjustment including insulin use, prior severe hypoglycemia, number of diabetic complications, and physical activity. However, the study participants were from one healthcare system, and the majority $(80 \%)$ were Caucasian.

Although the causes of hypoglycemia in patients with diabetes are multifactorial, there may be some explanations for the association of severe hypoglycemia with depression in patients with type 2 diabetes. First, the activation of the hypothalamic-pituitary-adrenal axis and sympathetic nervous system in depressed persons may affect the physiological response to hypoglycemia. ${ }^{25} 26$ Second, patients with dementia may have depressive symptoms, ${ }^{27}$ and are prone to developing severe hypoglycemia. ${ }^{28}$ Although we did not measure cognitive function at study entry, self-reported questionnaires including food frequency may exclude clinically evident dementia. Third, severe hypoglycemia per se may affect mood. Strachan et $a l^{29}$ reported that the elevation of depression scores persisted until 30 days after an episode of severe hypoglycemia in insulin-treated patients compared with those without severe hypoglycemia. It is conceivable that repeated exposure to episodes of severe hypoglycemia might induce depressive symptoms by contributing to the fear of hypoglycemia. ${ }^{30}$

This study confirmed clinical characteristics of depressed patients with diabetes similar to those reported in Western populations, that is, obesity, unhealthy lifestyles, poor glycemic control, and advanced diabetic complications. ${ }^{5-7}$ 22-24 However, a study in one Japanese hospital comprising 3305 participants failed to reveal that depressed patients with diabetes have a higher HbAlc or BMI than those who were not depressed. ${ }^{31}$ This disagreement may be due to the difference in study participants (eg, insulin users $41.6 \%$ vs $26.5 \%$ in our study). In this study, depressive symptoms were not associated with an increase in HS-CRP or HOMA2-IR, whereas low-grade systemic inflammation and insulin resistance have been observed in those with depression in Western populations. ${ }^{32}{ }^{33}$ In another Japanese study $(\mathrm{n}=3573$, mean BMI $24.5 \mathrm{~kg} / \mathrm{m}^{2}$ ), an increase in HS-CRP was not associated with depressive symptoms. ${ }^{34}$ This ethnic difference may be explained by differences in the BMI of patients with type 2 diabetes, in that Japanese patients with type 2 diabetes are typically non-obese.

A possible association between diet and depression has been reported, ${ }^{24}$ although the results are conflicting. In this study, sucrose intake increased according to the severity of depressive symptoms, while magnesium and folate intakes decreased. The increased incidence of hypoglycemia in depressed patients may lead to increased sucrose intake in an attempt to prevent hypoglycemic episodes. Reduced intakes of magnesium and folate may be due to a reduced intake of vegetables and 
Table 4 Severe hypoglycemia according to the CES-D score in Japanese patients with type 2 diabetes treated with sulfonylurea and/or insulin

\begin{tabular}{|c|c|c|c|c|c|}
\hline & \multicolumn{4}{|c|}{ CES-D score } & \multirow[b]{2}{*}{$\mathrm{p}$ for trend } \\
\hline & $\leq 9$ & 10-15 & $16-23$ & $\geq 24$ & \\
\hline $\mathrm{n}$ & 2141 & 362 & 172 & 81 & \\
\hline Severe hypoglycemia (\%) & $2.4 \%$ & $4.1 \%$ & $5.8 \%$ & $11.1 \%$ & $<0.0001$ \\
\hline $\begin{array}{l}\text { Multiple logistic regression analysis } \\
\text { for severe hypoglycemia* }\end{array}$ & Referent & 1.64 (0.86 to 2.94$)$ & 2.09 (0.96 to 4.12$)$ & 3.66 (1.53 to 7.99$) \dagger$ & $<0.001$ \\
\hline
\end{tabular}

Multivariate adjustments include age, sex, duration of diabetes, $\mathrm{HbA1c}$, insulin treatment, self-monitoring of blood glucose, leisure-time physical activity, skipping breakfast, dysesthesia of both feet, ischemic heart disease, and stroke.

${ }^{\star}$ Results are reported as ORs $(95 \% \mathrm{Cl})$.

$t p<0.01$ versus referent.

CES-D, Center for Epidemiologic Studies Depression Scale; HbA1c, glycated hemoglobin.

fruits. Since magnesium and folate are involved in neuronal activity, depression may be associated with low serum concentrations of magnesium ${ }^{35}$ and folate. ${ }^{36}$ Also, since most studies examining this issue are observational and cross-sectional, the causal relationship remains to be elucidated. Additionally, depressed people sometimes show characteristic eating behaviors such as skipping meals, especially breakfast. ${ }^{37}$ Such behavior was also observed in relation to the severity of depressive symptoms in this study (table 3 ).

A strength of this study is that it included a relatively large population of patients with type 2 diabetes not taking antidepressants and antipsychotic drugs. These medications may affect metabolic parameters. ${ }^{38}$ Furthermore, we examined many pathophysiological aspects of type 2 diabetes, including laboratory measurements and lifestyle factors.

A potential limitation of the study is that causal relationships cannot be addressed because of its crosssectional design. Second, we did not investigate the socioeconomic status of participants, including marital status, income, and education, as asking for information on these factors can sometimes be difficult in epidemiological studies in the Japanese cultural setting. ${ }^{31} 3439$ Third, severe hypoglycemia was self-reported. However, since half of the participants in the most depressed group performed self-monitoring of blood glucose, it was usually measured at episodes of hypoglycemia. Moreover, the retrospective recall of severe hypoglycemia has been shown to be robust for up to 1 year in patients with type 2 diabetes mellitus. ${ }^{40}$ Finally, the rates of severe hypoglycemia were generally low, particularly for insulin-treated patients with type 2 diabetes compared with those reported in other countries. ${ }^{41}$ Therefore, the applicability of the present findings to other populations remains to be elucidated.

In conclusion, this cross-sectional study revealed that severe hypoglycemia was positively associated with the severity of depressive symptoms in Japanese patients with type 2 diabetes independent of glycemic control, insulin therapy, lifestyle factors, and diabetic complications. Since severe hypoglycemia and depression are known risk factors for morbidity and mortality in patients with diabetes, clinicians should be aware of this combination in clinical settings.

Acknowledgements The authors thank Dr Yoichiro Hirakawa, Dr Yutaka Kiyohara, Dr Dongchon Kang, Dr Shuzo Kumagai, Dr Shigenobu Kanba (Kyushu University), Dr Nobutaka Tsutsu, Dr Nobuhiro Sasaki, Dr Sakae Nohara, Dr Chie Miyagawa, Dr Yuji Komorida (Fukuoka Red Cross Hospital), Dr Kiyohide Nunoi, Dr Yuichi Sato, Dr Hirofumi Imoto, Dr Masae Toyonaga, Dr Ayumi Yamauchi, Dr Chisa Matsumoto (St. Mary's Hospital), Dr Kazushi Amano, Dr Kaori Itoh (Steel Memorial Yawata Hospital), Dr Daisuke Gotoh, Dr Toshitaka Himeno, Dr Chie Sejima (Kyushu Central Hospital), Dr Noriyasu Shinohara, Dr Ayako Tsutsumi, Dr Akiko Sumi (Fukuoka Higashi Medical Center), Dr Masahiro Nakano, Dr Mina Matsuo, Dr Shoko Morimoto, Dr Tomoko Hyodo (Hakujyuji Hospital), Dr Masae Minami (Clinic Minami Masae), Dr Miya Wada (Wada Miya Naika Clinic), Dr Yoshifumi Yokomizo (Yokomizo Naika Clinic), Dr Masanori Kikuchi (Kikuchi Naika Clinic), Dr Riku Nomiyama (Suzuki Naika Clinic), Dr Shin Nakamura (Nakamura Naika Clinic), Dr Kenji Tashiro (Oshima Eye Hospital), Dr Mototaka Yoshinari (Yoshinari Naika Clinic), Dr Kojiro Ichikawa (Fukutsu Naika Clinic), and Dr Teruo Omae (Hisayama Research Institute For Lifestyle Diseases), clinical research coordinators; Chiho Ohba, Yumi Ono (Hisayama Research Institute For Lifestyle Diseases), Kayoko Sekioka (Kyushu University), and administration office; Tomoko Matake (Hisayama Research Institute For Lifestyle Diseases), and Junko Ishimatsu (Kyushu University).

Contributors $\mathrm{MI}$ and $\mathrm{HF}$ were responsible for the study concept and design. YK and MI conducted the analyses, and TO, SK, HI, TJ, YI, UN, and TK helped with interpreting the data and contributed to the discussion. MI and YK drafted the manuscript, and all authors participated in revising the manuscript critically and approved the final version. $\mathrm{Ml}$ is the guarantor and, as such, had full access to all the data in the study. He takes responsibility for the integrity of the data and the accuracy of the data analysis.

Funding This study was supported in part by Grants-in-Aid for Scientific Research (no. 23249037 and no.23659353) for MI from the Ministry of Education, Culture, Sports, Science and Technology of Japan.

Competing interests None declared.

Patient consent Obtained.

Ethics approval Obtained.

Provenance and peer review Not commissioned; externally peer reviewed.

Data sharing statement No additional data are available.

Open Access This is an Open Access article distributed in accordance with the Creative Commons Attribution Non Commercial (CC BY-NC 4.0) license, which permits others to distribute, remix, adapt, build upon this work noncommercially, and license their derivative works on different terms, provided the original work is properly cited and the use is non-commercial. See: http:// creativecommons.org/licenses/by-nc/4.0/ 


\section{REFERENCES}

1. Anderson RJ, Freedland KE, Clouse RE, et al. The prevalence of comorbid depression in adults with diabetes: a meta-analysis. Diabetes Care 2001;24:1069-78.

2. Mezuk B, Eaton WW, Albrecht S, et al. Depression and type 2 diabetes over the lifespan: a meta-analysis. Diabetes Care 2008;31:2383-90.

3. Golden SH, Lazo M, Carnethon M, et al. Examining a bidirectional association between depressive symptoms and diabetes. JAMA 2008;299:2751-9.

4. Pan A, Lucas $M$, Sun $Q$, et al. Bidirectional association between depression and type 2 diabetes mellitus in women. Arch Intern Med 2010;170:1884-91.

5. Lustman PJ, Anderson RJ, Freedland KE, et al. Depression and poor glycemic control: a meta-analytic review of the literature. Diabetes Care 2000;23:934-42.

6. de Groot M, Anderson R, Freedland KE, et al. Association of depression and diabetes complications: a meta-analysis. Psychosom Med 2001;63:619-30.

7. Egede LE, Ellis C. Diabetes and depression: global perspectives. Diabetes Res Clin Pract 2010;87:302-12.

8. Lloyd CE, Roy T, Nouwen A, et al. Epidemiology of depression in diabetes: international and cross-cultural issues. J Affect Disord 2012;142(Suppl):S22-9.

9. Chan JC, Malik V, Jia W, et al. Diabetes in Asia: epidemiology, risk factors, and pathophysiology. JAMA 2009;301:2129-40.

10. WHO World Health Organization. Preventing suicide: a global imperative. 2014. http://www.who.int/mental health/suicideprevention/world_report_2014/en/ (accessed 15 Sep 2014).

11. Ohkuma $T$, Fujii $\bar{H}$, Iwase $M$, et al. Impact of eating rate on obesity and cardiovascular risk factors according to glucose tolerance status: the Fukuoka Diabetes Registry and the Hisayama Study. Diabetologia 2013:56:70-7.

12. Examination Committee of Criteria for Obesity Disease in Japan, Japan Society for the Study of Obesity. New criteria for obesity disease in Japan. Circ $J$ 2002;66:987-92.

13. Ainsworth BE, Haskell WL, Whitt MC, et al. Compendium of physical activities: an update of activity codes and MET intensities. Med Sci Sports Exerc 2000;32:S498-504.

14. Kobayashi S, Honda S, Murakami K, et al. Both comprehensive and brief self-administered diet history questionnaires satisfactorily rank nutrient intakes in Japanese adults. J Epidemiol 2012;22:151-9.

15. Radloff LS. The CES-D scale: a self-report depression scale for research in the general population. Appl Psychol Meas 1977;1:385-401.

16. Fava GA, Pilowsky I, Pierfederici A, et al. Depression and illness behavior in a general hospital: a prevalence study. Psychother Psychosom 1982;38:141-53.

17. Matsuo S, Imai E, Horio M, et al., Collaborators developing the Japanese equation for estimated GFR. Revised equations for estimated GFR from serum creatinine in Japan. Am $J$ Kidney Dis 2009:53:982-92.

18. Levey AS, de Jong PE, Coresh J, et al. The definition, classification, and prognosis of chronic kidney disease: a KDIGO controversies conference report. Kidney Int 2011;80:17-28.

19. Alberti KG, Eckel RH, Grundy SM, et al. Harmonizing the metabolic syndrome: a joint interim statement of the International diabetes federation task force on epidemiology and prevention; National heart, lung, and blood institute; American heart association; World heart federation; International atherosclerosis society; and International association for the study of obesity. Circulation 2009;120:1640-5.

20. Homa calculator. http://www.dtu.ox.ac.uk (accessed Jun 2012).

21. Zoungas S, Patel A, Chalmers J, et al., ADVANCE Collaborative Group. Severe hypoglycemia and risks of vascular events and death. N Engl J Med 2010;363:1410-18.

22. Katon W, von Korff M, Ciechanowski $P$, et al. Behavioral and clinical factors associated with depression among individuals with diabetes. Diabetes Care 2004;27:914-20.
23. Lysy Z, Da Costa D, Dasgupta K. The association of physical activity and depression in type 2 diabetes. Diabet Med 2008;25: 1133-41.

24. Lai JS, Hiles S, Bisquera A, et al. A systematic review and meta-analysis of dietary patterns and depression in community-dwelling adults. Am J Clin Nutr 2014;99:181-97.

25. Green AJ, Fox KM, Grandy S; SHIELD Study Group. Self-reported hypoglycemia and impact on quality of life and depression among adults with type 2 diabetes mellitus. Diabetes Res Clin Pract 2012;96:313-18.

26. Katon WJ, Young BA, Russo J, et al. Association of depression with increased risk of severe hypoglycemic episodes in patients with diabetes. Ann Fam Med 2013;245-50.

27. Katon WJ, Lin EH, Williams LH, et al. Comorbid depression is associated with an increased risk of dementia diagnosis in patients with diabetes: a prospective cohort study. J Gen Intern Med 2010;25:423-9.

28. Yaffe K, Falvey CM, Hamilton N, et al., Health ABC Study. Association between hypoglycemia and dementia in a biracial cohort of older adults with diabetes mellitus. JAMA Intern Med 2013;173:1300-6.

29. Strachan MW, Deary IJ, Ewing FM, et al. Recovery of cognitive function and mood after severe hypoglycemia in adults with insulin-treated diabetes. Diabetes Care 2000;23:305-12.

30. Sheu WH, Ji LN, Nitiyanant W, et al. Hypoglycemia is associated with increased worry and lower quality of life among patients with type 2 diabetes treated with oral antihyperglycemic agents in the Asia-Pacific region. Diabetes Res Clin Pract 2012;96: 141-8.

31. Tsujii S, Hayashino Y, Ishii H; Diabetes Distress and Care Registry at Tenri Study Group. Diabetes distress, but not depressive symptoms, is associated with glycaemic control among Japanese patients with type 2 diabetes: Diabetes Distress and Care Registry at Tenri (DDCRT 1). Diabet Med 2012;29:1451-5.

32. Stuart MJ, Baune BT. Depression and type 2 diabetes: inflammatory mechanisms of a psychoneuroendocrine co-morbidity. Neurosci Biobehav Rev 2012;36:658-76.

33. Kan C, Silva N, Golden $\mathrm{SH}$, et al. A systematic review and meta-analysis of the association between depression and insulin resistance. Diabetes Care 2013;36:480-9.

34. Hayashino Y, Mashitani T, Tsujii S, et al., Diabetes Distress and Care Registry at Tenri Study Group. Elevated levels of hs-CRP are associated with high prevalence of depression in Japanese patients with type 2 diabetes: The Diabetes Distress and Care Registry at Tenri (DDCRT 6). Diabetes Care 2014;37:2459-65.

35. Huang JH, Lu YF, Cheng FC, et al. Correlation of magnesium intake with metabolic parameters, depression and physical activity in elderly type 2 diabetes patients: a cross-sectional study. Nutr $J$ 2012;11:41.

36. Murakami K, Mizoue T, Sasaki S, et al. Dietary intake of folate, other B vitamins, and omega-3 polyunsaturated fatty acids in relation to depressive symptoms in Japanese adults. Nutrition 2008;24:140-7.

37. Fulkerson JA, Sherwood NE, Perry CL, et al. Depressive symptoms and adolescent eating and health behaviors: a multifaceted view in a population-based sample. Prev Med 2004;38:865-75.

38. Pan A, Sun Q, Okereke Ol, et al. Use of antidepressant medication and risk of type 2 diabetes: results from three cohorts of US adults. Diabetologia 2012;55:63-72.

39. Sekita A, Arima H, Ninomiya T, et al. Elevated depressive symptoms in metabolic syndrome in a general population of Japanese men: a cross-sectional study. BMC Public Health 2013;13:862.

40. Akram K, Pedersen-Bjergaard U, Carstensen B, et al. Prospective and retrospective recording of severe hypoglycaemia, and assessment of hypoglycaemia awareness in insulin-treated type 2 diabetes (Letter). Diabet Med 2009;26:1306-8.

41. UK Hypoglycaemia Study Group. Risk of hypoglycaemia in types 1 and 2 diabetes: effects of treatment modalities and their duration. Diabetologia 2007;50:1140-7. 